\title{
As Estruturas Multiplicativas e a formação de professores que ensinam Matemática na Bahia: um projeto de larga escala
}

\author{
Sandra Maria Pinto Magina \\ Eurivalda Ribeiro dos Santos Santana \\ Irene Mauricio Cazorla
}

\section{Resumo}

Este artigo apresenta o projeto de pesquisa sobre as estruturas multiplicativas (PEM - FAPESB, PESoo19/2013), desenvolvido em rede por grupos pesquisadores de cinco universidades baianas (UESC, UESB, UNEB, UEFS, UFRB) e o grupo EmFoco. O objetivo do projeto foi promover uma formação de professores que permitisse desenvolver estratégias de ensino que possibilitassem a expansão e apropriação do campo conceitual multiplicativo pelos estudantes. A pesquisa envolveu dois estudos, um diagnóstico ancorado na Teoria dos Campos Conceituais e no esquema construído por Magina, Santos e Merlini para classificação dos problemas multiplicativos. O instrumento diagnóstico foi aplicado a 4.076 estudantes baianos do Ensino Fundamental. Os resultados permitiram traçar um panorama das competências dos estudantes e subsidiaram o segundo estudo, que propiciou a formação de professores desses estudantes. Este foi realizado ancorado no modelo metodológico de formação intitulado Espiral RePARe 'reflexão-planejamento-ação-reflexão' - elaborada por MAGINA (2008), o qual tem uma vertente reflexiva sobre elementos que compõem o processo de ensino. Esse trabalho, realizado com os professores no lócus da escola, a partir da reflexão sobre os resultados do diagnóstico dos estudantes e do trabalho desenvolvido em sala de aula, indica que o modelo RePARe pode ser usado no ensino de diferentes conteúdos matemáticos e nos vários níveis de ensino.

Palavras-chave: Estruturas Multiplicativas. Teoria dos Campos Conceituais. Formação de Professes. Metodologia RePARe. 


\title{
Multiplicative structures and in service program for those who teach Mathematics in Bahia: a large scale project
}

\author{
Sandra Maria Pinto Magina \\ Eurivalda Ribeiro dos Santos Santana \\ Irene Mauricio Cazorla
}

Abstract

his article presents the research project on multiplicative structures (PEM - FAPESB, PESo019 / 2013), developed in a network by research groups from five Brazilian universities (UESC, UESB, UNEB, UEFS, UFRB) and the EmFoco group. The objective of the project was to promote a teacher education that allowed the development of teaching strategies that allowed the expansion and appropriation of the multiplicative conceptual field by the students. The research involved two studies, a diagnosis anchored in Conceptual Field Theory and in the scheme constructed by Magina, Santos and Merlini to classify multiplicative problems. The diagnostic instrument was applied to 4,076 Bahian students from Elementary School. The results allowed to draw a panorama of the competences of the students and subsidized the second study, which provided the training of teachers of these students. This was carried out anchored in the methodological model of formation called Espiral RePARe - 'reflection-planning-action-reflection' - elaborated by MAGINA (2008), which has a reflexive slope on elements that make up the teaching process. This work, carried out with the teachers at the school site, from the reflection on the results of the students' diagnosis and the work done in the classroom, indicates that the RePARe model can be used in the teaching of different mathematical contents and at various levels education

Keywords: Multiplicative Structures. Conceptual Field Theory. Teacher Training. RePARe methodology. 


\section{Introdução}

Este artigo apresenta o projeto de pesquisa "As estruturas multiplicativas e a formação de professores que ensinam matemática na Bahia - PEM” (MAGINA, 2013), financiado pela Fundação de Amparo à Pesquisa do Estado da Bahia (FAPESB), cujos resultados serviram de base para a apresentação dos outros cinco artigos deste número especial. Ele foi desenvolvido em rede, envolvendo seis núcleos de pesquisas na Bahia, a saber: Universidade Estadual de Santa Cruz (núcleo proponente); Universidade Estadual do Sudoeste da Bahia - Campus Vitória da Conquista (núcleo UESB-Conquista); Universidade do Estado da Bahia, Campus Senhor do Bonfim (núcleo UNEB-Bonfim); Universidade Estadual de Feira de Santana (núcleo UEFS-Feira) em parceria com o Núcleo de Educação Matemática de Feira de Santana-NEEMFS; Universidade Federal do Recôncavo da Bahia (núcleo UFRB-Amargosa) e Grupo Educação Matemática em Foco (núcleo EMFoco-Salvador). Cada núcleo, por sua vez, trabalhou em parceria com, pelo menos, uma escola pública.

O objetivo principal desse projeto de pesquisa foi promover o desenvolvimento de estratégias de ensino que possibilitassem a expansão e apropriação do campo conceitual multiplicativo (VERGNAUD, 1983, 1988, 1996) pelos estudantes. Para tanto o projeto envolveu dois estudos. O primeiro estudo $(\alpha)$ focou o diagnostico do desempenho e estratégias dos estudantes do Ensino Fundamental a partir de um instrumento contendo 14 situações do campo multiplicativo. O segundo estudo $(\beta)$ focou a formação dos professores dos estudantes que responderam o instrumento do primeiro estudo.

A pesquisa PEM foi ancorada na Teoria dos Campos Conceituais proposta por Vergnaud (1983, 1988, 1994, 2009) e em sua releitura, no que tange às Estruturas multiplicativas feita por Magina, Santos e Merlini (2010) e, metodologicamente em um modelo de formação de professores na espiral RePARe - 'reflexão-planejamento-ação-reflexão' proposto por (MAGINA 2008), visando em última instância, a promoção e o desenvolvimento de estratégias de ensino que possibilitem aos professores a expansão e apropriação deste campo conceitual pelos estudantes.

Antes de iniciarmos uma descrição mais detalhada de cada um desses estudos pertencentes ao projeto, historiamos o percurso de construção desse projeto.

\section{Os projetos que precederam e subsidiaram o PEM}

Dois projetos, um realizado em São Paulo e outro na Bahia, foram os precursores do PEM e deles emergiram duas de suas características mais importantes. Descreveremos cada um desses projetos, apontando a contribuição de cada um deles para compor o PEM.

O projeto "(Re)significar as estruturas multiplicativas a partir da formação 'reflexãoação-planejamento-reflexão’ do professor” coordenado por Magina (2008) foi realizado entre ISSN 2526-2882 
2008 e 2010, com suporte do Conselho Nacional de Desenvolvimento Científico e Tecnológico (CNPq). Teve como objetivo desenvolver uma formação continuada de professores de caráter colaborativa, na direção de testar a eficácia do modelo metodológico intitulado Espiral RePARe - 'reflexão-planejamento-ação-reflexão' - que promovesse o desenvolvimento de estratégias de ensino por parte do professor e que, consequentemente, possibilitasse a expansão e apropriação do Campo Conceitual das estruturas multiplicativas por seus estudantes.

Para atingir esse propósito, foram desenvolvidos dois estudos. O primeiro (Estudo $\alpha$ ), de cunho descritivo voltou-se para identificar as competências dos estudantes; enquanto que o segundo (Estudo $\beta$ ) focou a identificação das concepções e crenças dos professores sobre o ensino do campo multiplicativo.

Os resultados obtidos no Estudo $\alpha$ serviram de subsídio para a realização do Estudo $\beta$, que envolveu uma formação de professores. Esta formação iniciou justamente pela reflexão coletiva com os professores, sobre o desempenho e estratégias de ação dos estudantes que responderam o instrumento diagnóstico (ação diagnóstica inicial). Nos momentos de reflexão coletiva, buscava-se identificar as crenças apresentadas por esses professores tanto sobre as estruturas multiplicativas, quanto o seu ensino. Na esteira dessa reflexão, que vinha acompanhada de uma discussão teórica sobre esse campo conceitual, vinha o planejamento coletivo de duas atividades, cuja ação acontecia nas salas de aula dos professores em formação.

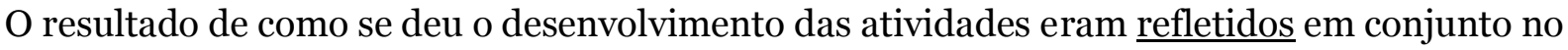
encontro seguinte da formação. Assim, fica claro que o cerne dessa dinâmica formativa era o trabalho coletivo, culminando com uma produção colaborativa de alternativas metodológicas e estratégias de ensino registradas em portfólios. O Modelo de formação RePARe será mais bem discutido adiante, quando estivermos expondo com detalhes o Estudo $\beta$.

O projeto "Um estudo sobre o domínio das Estruturas Aditivas nas séries iniciais do Ensino Fundamental no Estado da Bahia" e cognominado por "Pesquisa das Estruturas Aditivas" (PEA), foi financiado pela Fundação de Amparo à Pesquisa do Estado da Bahia (FAPESB). O projeto desenvolveu-se dentro de uma rede colaborativa entre pesquisadores de universidades estaduais da Bahia, professores de escolas públicas baianas e oito Núcleos da Sociedade Brasileira de Educação Matemática, Regional Bahia (SBEM/BA), apresentados no Quadro 1.

A Rede PEA teve como finalidade principal desenvolver uma formação colaborativa com professores da Escola Básica e pesquisadores de universidades baianas, visando à construção de tarefas, a serem implementadas na sala de aula, que contribuíssem para sanar dificuldades no ensino e na aprendizagem da adição e da subtração por estudantes dos anos iniciais do Ensino Fundamental. Para alcançar tal objetivo, a equipe realizou dois estudos entre 
2009 e 2010. No primeiro elaborou e aplicou um instrumento diagnóstico nas oito regiões da Bahia. Esse instrumento, elaborado por Santana (2010), era composto de 18 situaçõesproblema de adição e de subtração, todas utilizando apenas números naturais e não ultrapassando o total de 20 unidades. Esse instrumento foi aplicado em 5.807 estudantes do $2^{\circ}$ ao $5^{\circ}$ ano do Ensino Fundamental de 70 escolas distribuídas em 47 municípios baianos.

O segundo estudo, foi desenvolvido por apenas sete núcleos, tratou-se de uma formação colaborativa envolvendo os pesquisadores e os professores das escolas que participaram da pesquisa em 2009, tomando como base metodológica a Espiral RePARe (MAGINA, 2008). Foram ao todo 280 professores formandos.

Quadro 1 - Núcleos integrantes da Rede PEA por região

\begin{tabular}{|c|c|c|}
\hline Núcleo & $\begin{array}{l}\text { Universidade / } \\
\text { Educação Básica }\end{array}$ & Região da Bahia \\
\hline Núcleo de Amargosa & $\begin{array}{l}\text { Universidade Federal do } \\
\text { Recôncavo da Bahia (UFRB) }\end{array}$ & $\begin{array}{l}\text { Recôncavo Baiano, com sede em } \\
\text { Amargosa }\end{array}$ \\
\hline Núcleo de Barreiras & $\begin{array}{lll}\text { Universidade } & \text { Federal da } \\
\text { Bahia (UFBA) } & & \end{array}$ & $\begin{array}{l}\text { Região Oeste, com sede em } \\
\text { Barreiras }\end{array}$ \\
\hline 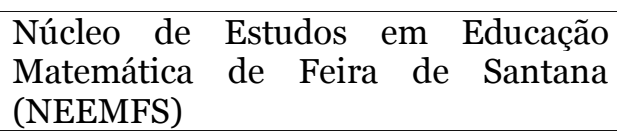 & $\begin{array}{l}\text { Professores da Educação } \\
\text { Básica }\end{array}$ & $\begin{array}{l}\text { Região Nordeste } \\
\text { Feijão, com sede em Feira de } \\
\text { Santana }\end{array}$ \\
\hline $\begin{array}{l}\text { Núcleo de Ilhéus com o Grupo de } \\
\text { Pesquisa em Educação Matemática, } \\
\text { Estatística e em Ciências (GPEMEC) }\end{array}$ & $\begin{array}{l}\text { Universidade Estadual de } \\
\text { Santa Cruz (UESC) }\end{array}$ & Região Sul, com sede em Ilhéus \\
\hline $\begin{array}{l}\text { Núcleo de Produção e Divulgação do } \\
\text { Conhecimento } \quad \text { em } \\
\text { Matemática (NPDCED) }\end{array}$ & $\begin{array}{l}\text { Universidade Estadual do } \\
\text { Sudoeste da Bahia (UESB) }\end{array}$ & $\begin{array}{l}\text { Região Sudoeste Jequié, com sede } \\
\text { em Jequié }\end{array}$ \\
\hline Núcleo EMFoco & $\begin{array}{l}\text { Professores da Educação } \\
\text { Básica }\end{array}$ & $\begin{array}{l}\text { Região Metropolitana, com sede em } \\
\text { Salvador }\end{array}$ \\
\hline Núcleo de Senhor do Bonfim & $\begin{array}{l}\text { Universidade do Estado da } \\
\text { Bahia (UNEB). Campus VII }\end{array}$ & $\begin{array}{l}\text { Região Norte, com sede em Senhor } \\
\text { do Bonfim }\end{array}$ \\
\hline $\begin{array}{l}\text { Núcleo de Conquista na UESB - GEEM } \\
\text { - Grupo de Estudo em Educação } \\
\text { Matemática }\end{array}$ & $\begin{array}{l}\text { Universidade Estadual do } \\
\text { Sudoeste da Bahia (UESB) }\end{array}$ & $\begin{array}{l}\text { Na Região Sudoeste, Planalto da } \\
\text { Conquista, com sede em Vitória da } \\
\text { Conquista }\end{array}$ \\
\hline
\end{tabular}

Fonte: Dados do PEA de 2009-2010.

Para a realização das ações de pesquisa, a Rede PEA manteve uma dinâmica de grupo operativo para estudar a teoria que embasava essas ações. Assim, no seio de cada Núcleo havia reuniões presenciais semanais com a equipe e a Rede, representada pelos coordenadores de cada Núcleo e alguns de seus pesquisadores, reuniam-se presencialmente a cada seis meses.

Desta forma, o referencial teórico foi efetivamente fomentado e apropriado pelos coordenadores nas três primeiras reuniões presenciais da Rede PEA, ficando cada um deles responsáveis, por meio das constantes reuniões semanais de seu núcleo, por trabalhar essa apropriação com sua equipe. As reuniões presenciais da Rede, também, subsidiaram a construção dos instrumentos de pesquisa, o planejamento do processo formativo e, a análise e discussão dos resultados que estavam sendo levantados por cada Núcleo. Ao final da formação 
dos professores, cada Núcleo realizou um seminário, com o objetivo de apresentar os resultados da pesquisa realizada em sua região e, teve a participação de secretários municipais de educação, professores da rede municipal e estadual, membros da comunidade acadêmica, estudantes dos cursos de Licenciatura em Pedagogia e em Matemática.

O quarto encontro presencial da Rede PEA, teve como objetivo principal, discutir os principais resultados da pesquisa e realizar o lançamento de quatro cadernos de estudos destinados aos professores dos anos iniciais e elaborados a partir das experiências oriundas do processo formativo de cada um dos sete Núcleos da Rede PEA (SANTANA; CORREIA, 2011). Os cadernos foram elaborados numa parceria entre os pesquisadores dos sete Núcleos.

Para além da publicação dos quatro cadernos de estudos, a Rede PEA publicou seus resultados em revistas científicas e anais de eventos da área de Educação Matemática.

Tendo descrito os dois projetos que serviram de base de sustentação do PEM, apresentaremos a seguir a teoria que ancorou os projetos, qual seja a Teoria dos Campos Conceituais. Deter-nos-emos em especial no campo conceitual multiplicativo ou, simplesmente, estrutura multiplicativa.

\section{O Campo Conceitual Multiplicativo: uma visão resumida}

Muitos pesquisadores têm apresentado em seus estudos o campo conceitual multiplicativo (VERGNAUD, 1983, 1988, 1994; MORO, 2005; MAGINA, 2013; GITIRANA, ET. AL, 2013; SANTOS, 2014; MAGINA, SANTOS, MERLINI, 2010, 2014, 2016; SANTANA, LAUTERT, FILHO, 2017, entre muitos outros). Aqui, apresentamos uma breve explicação da Teoria e, explanamos um pouco mais a respeito do esquema proposto por Magina, Santos e Merlini $(2014,2016)$ para classificação dos tipos de problemas multiplicativos. É importante esclarecer que tal classificação atende apenas às situações com transformações lineares.

Dos pressupostos presentes na TCC compartilhamos da premissa posta por essa teoria de que o conhecimento emerge a partir da resolução de problemas, em que seu surgimento perpassa pela ação do sujeito sobre a situação. Nesse contexto, é preciso que essa ação venha acompanhada de uma reflexão, para que não se torne apenas uma competência adquirida, mas sim, que se encaminhe na direção da formação e desenvolvimento do conceito. Assim, para formar um conceito é necessário que o sujeito interaja com ele por meio de uma diversidade de situações. Isto porque uma situação, por mais simples que ela se apresente, traz em seu bojo vários conceitos (VERGNAUD, 1994, 1998, 2009).

Nessa teoria são as situações que dão significado ao conceito. Isto significa que quanto maior o número de situações diferentes forem experenciadas pelo sujeito, mais amplo o significado desse conceito. Além disso, é por meio da representação simbólica que o estudante se expressa sobre o conceito dentro da situação, relacionando o significado da situação com 
suas propriedades. Essas últimas, por sua vez, podem estar explícitas ou implícitas para o estudante.

Foi com essas premissas básicas da TCC que Magina, Santos e Merlini (2012) elaboraram um esquema para classificar os problemas multiplicativos dentro de dois tipos de relações: quaternária e ternária.

\section{As relações quaternárias}

As relações quaternárias estão presentes em uma situação-problema quando esta envolve uma dupla relação entre duas quantidades de naturezas distintas. Nesta relação temos três eixos: proporção simples, proporção dupla e proporção múltipla, sendo que todos esses eixos podem ter duas classes: "um para muitos" e "muito para muitos". A seguir apresentamos um exemplo de proporção simples, um para muitos.

Exemplo 1: CADA CADEIRA, QUE ESTÁ EM VOLTA DA MESA DE JANTAR DE JOÃO, TEM 4 PERNAS. SE JoÃo TIVER 12 CADEIRAS EM VOLTA DESSA MESA, QUANTAS PERNAS DE CADEIRAS TERÃO EM VOLTA DESSA MESA?

$\begin{array}{cc}\text { QuANTIDADE DE } & \text { QuanTIDADE DE } \\ \text { CADEIRAS } & \text { PERnAS }\end{array}$

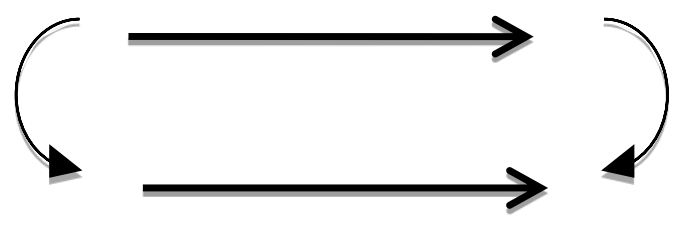

No Exemplo 1 se conhece três valores e se pergunta pelo quarto, envolvendo assim uma relação quaternária no âmbito do eixo proporção simples, dentro da classe de um para muitos, sendo que o tipo de quantidade envolvida é discreta. As setas presentes no esquema de resolução indicam duas maneiras possíveis de resolver a situação: (1) por meio de escalar multiplicativo, em que se estabelece a relação proporcional entre as quantidades de cadeiras e aplica esta mesma proporção para as quantidades de pernas de cadeiras; ou (2) por meio do estabelecimento de relação funcional, estabelecida entre o número de cadeira e o número de pernas de cadeira.

No Exemplo 2 observamos uma situação com uma relação quaternária do eixo proporção simples, classe de um para muitos. 
Exemplo 2: A CADA TRÊS VOLTAS QUE LUANA DÁ NO BRINQUEDO DO PARQUE DE DIVERSÕES ELA PAGA $\mathrm{R} \$ 5$,OO. SE ELA DER 12 VOLTAS QUANTO VAI PAGAR?

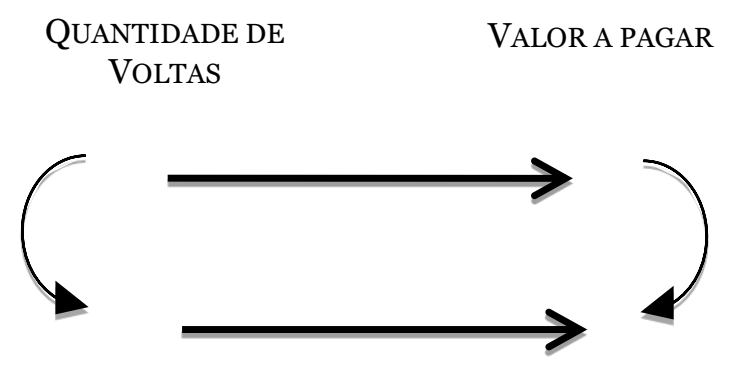

No Exemplo 2 são dados três valores e se pergunta pelo quarto, estabelecendo uma relação quaternária do eixo proporção simples, se configurando classe de muitos para muitos, pois a relação não é unitária, uma vez que para cada três voltas se paga cinco reais. O escalar multiplicativo (as setas verticais) estabelece a relação proporcional entre as quantidades de voltas e, essa mesma, relação proporcional é mantida para a relação valor a pagar. A relação funcional (as setas horizontais), estabelecida entre a quantidade de voltas e o valor a pagar e mantida quando a quantidade de voltas aumenta. Essas duas relações (escalar multiplicativo e relação funcional) são possibilidades de resolução da situaçãoproblema.

No eixo da proporção dupla são classificadas as situações que envolvem mais de duas grandezas e se configura numa relação quaternária, vejamos o Exemplo 5, da classe um para muitos.

Exemplo 3: UMA CRIANÇA BEBE, EM MÉDIA, 600 ML DE LEITE EM DOIS DIAS. QUANTO DE LEITE SERÁ CONSUMIDO POR TRÊS CRIANÇAS DA MESMA FAIXA ETÁRIA, EM 12 DIAS?

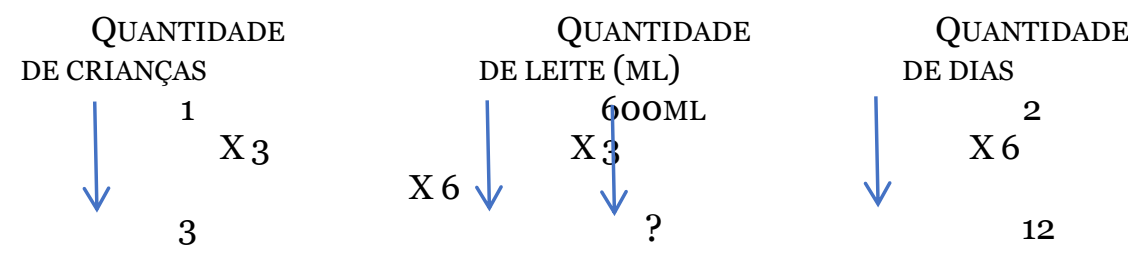

Para a resolução fixamos a grandeza (Quantidade de leite) que é proporcional às outras duas e, comparamos com cada uma separadamente. Primeiro, vamos comparar com a quantidade de crianças. 


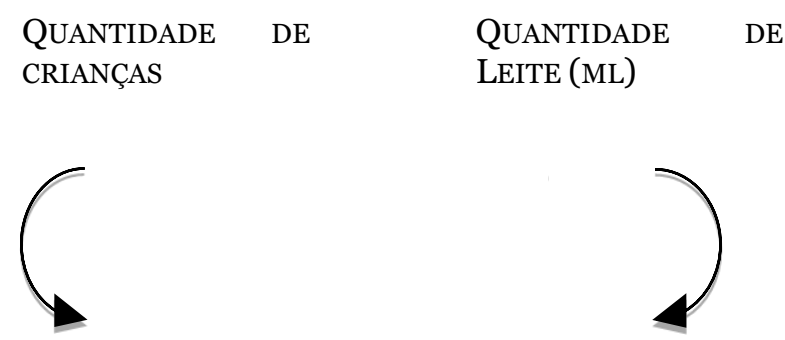

Dessa proporcionalidade temos $600 \mathrm{ml} \times 3=1.800 \mathrm{ml}$.

Em seguida, comparamos a grandeza quantidade de leite com a outra grandeza (Quantidade de dias).

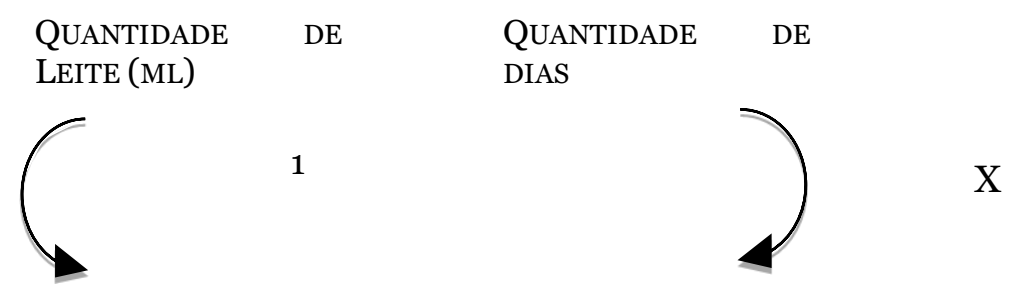

Na segunda proporcionalidade temos $1.800 \mathrm{ml} \mathrm{x} 6=10800 \mathrm{ml}$. Assim, as três crianças irão consumir $10.800 \mathrm{ml}$ em 12 dias.

A proporção múltipla se caracteriza por envolver mais de duas grandezas com proporção simples que são encadeadas, vejamos o Exemplo 4.

Exemplo 4: UMA FÁbRICA VENDE FARDOS DE PALHA DE AÇO COM PACOTES. EM UM FARDO, HÁ 14 PACOTES. CADA PACOTE CONTÉM O8 UNIDADES DE PALHA DE AÇO. SE O GERENTE DE UM RESTAURANTE COMPRAR 5 FARDOS, QUANTAS UNIDADES DE PALHA DE AÇO VAI LEVAR?

$\begin{array}{lllll}\begin{array}{l}\text { QUANTIDADE } \\ \text { FARDOS }\end{array} & \begin{array}{l}\text { QUANTIDADE } \\ \text { PACOTES }\end{array} & \text { DE } & \begin{array}{l}\text { QUANTIDADE } \\ \text { UNIDADES }\end{array}\end{array}$

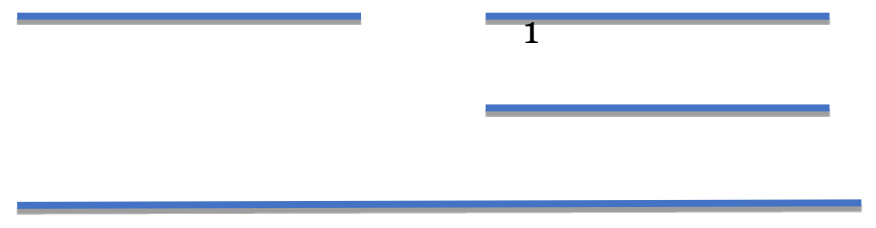

Para resolver a situação do Exemplo 4, podemos resolver duas proporções simples. Assim, para determinar a quantidade unidades de palha de aço em cada fardo, é necessário determinar a quantidade de palha de aço que há em cada pacote. 


$\begin{array}{lll}\text { QUANTIDADE } & \text { DE } & \text { QUANTIDADE } \\ \text { PACOTES } & & \text { UNIDADES }\end{array}$

$x=8$ unidades $\times 14=112$ unidades

Determinamos que em 14 pacotes há 112 unidades. Assim, precisamos encontrar a quantidade de unidades nos cinco fardos.

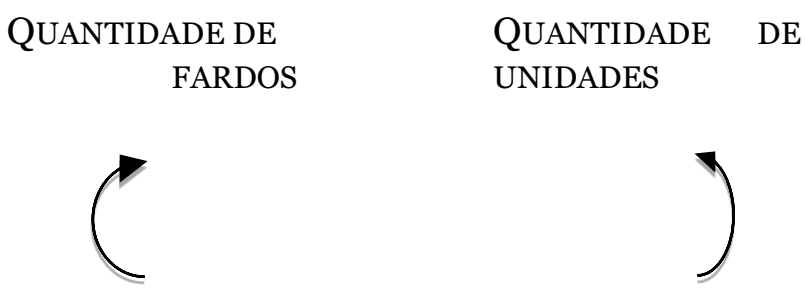

$\mathrm{y}=112$ unidades $\mathrm{x} 5=560$ unidades

Encontramos que em cinco fardos há 560 unidades.

O Exemplo 4 é uma situação de proporção múltipla da classe um para muitos, pois a relação unitária está explícita.

\section{As relações ternárias}

No que tange à relação ternária, apresentaremos dois exemplos (5A e 5B), sendo um envolvendo a comparação multiplicativa e o outro o produto de medida. Acreditamos que eles ajudarão a entender do que trata essa relação.

Nos dois exemplos são oferecidas duas quantidades e se pergunta pela terceira, caracterizando assim uma relação ternária. No caso do Exemplo 5A há uma comparação entre duas quantidades, cuja classe é a do referido desconhecido, visto que se conhece a quantidade do referente (que é a do João), a relação entre as quantidades de João e de Pedro (3 vezes mais) e pergunta-se pela quantidade de Pedro, que se conhecerá a partir da de João. As quantidades envolvidas na situação são discretas.

O Exemplo 5B, igualmente, envolve uma relação ternária, em que se estabelece um produto de medidas linear (o produto do comprimento da frente do terreno pelo comprimento do seu lado), numa configuração retangular, com a situação apresentando um tipo de quantidade contínua. 


Exemplo 5 A
JOÃO E PEDRO FAZEM COLEÇÃO DE

FIGURINHAS. JOÃO TEM 28 FIGURINHAS E PEDRO TEM 3 VEZES MAIS FIGURINHAS QUE JOÃO. QUANTAS FIGURINHAS PEDRO TEM?

\section{REFERIDO:}

QUANTIDADES DAS

FIGURINHAS DO PEDRO

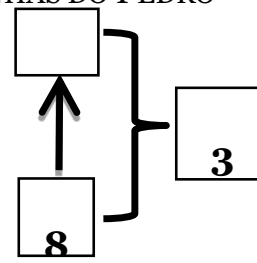

REFERENTE:

QUANTIDADE

DAS FIGURINHAS DO JOÃO
RELAĈ̣̃o

ENTRE

AS

DE JOÃO E

PEDRO

\section{Exemplo 5B}

JOÃO COMPROU UM TERRENO QUE TEM A FORMA DE RETÂNGULO. ELE MEDE 15 METROS DE FRENTE E 10 METROS DE LADO. QUAL A ÁREA DO TERRENO DE JOÃO?

A Figura 1, a seguir, apresenta o esquema proposto por Magina, Santos e Merlini (2012, 2014, 2016), no qual as situações-problema anteriores são exemplos que se enquadram nele e a partir do qual foram construídas as 14 situações-problema do instrumento diagnóstico que foi aplicado aos estudantes.

Figura 1: Esquema do Campo Conceitual Multiplicativo

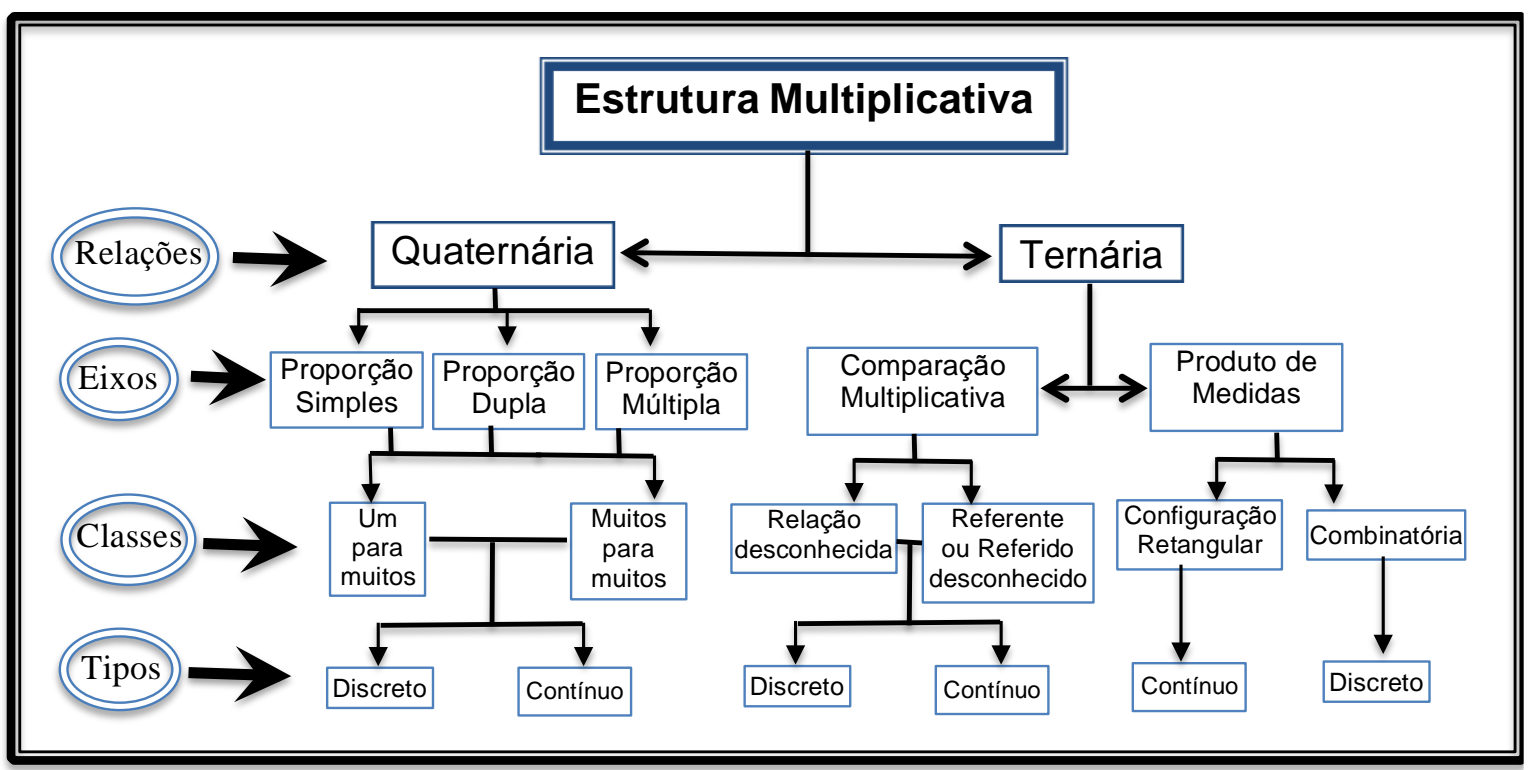

Fonte: Magina, Santos, Merlini, 2012, ajustado em 2016. 


\section{Discrição do PEM}

Como dito anteriormente, o PEM teve por objeto matemático o campo conceitual multiplicativo, o qual foi pesquisado no âmbito de dois estudos: o estudo diagnóstico direcionado para estudantes do Ensino Fundamental (Estudo $\alpha$ ) e a formação continuada do professor (Estudo $\beta$ ). Descreveremos a seguir cada um dos estudos.

O Estudo $\alpha$ foi de cunho descritivo, em que foi aplicado um instrumento diagnóstico a 4.076 estudantes baianos do Ensino Fundamental (EF), assim distribuídos: 408 estudantes do $1^{\circ}$ ano, 371 do $2^{\circ}$ ano, 630 do $3^{\circ}$ ano, 535 do $4^{\circ}$ ano, 689 do $5^{\circ}$ ano, 411 do $6^{\circ}$ ano, 433 do $7^{\circ}$, 331 do $8^{\circ}$ ano e 267 do $9^{\circ}$ ano. A aplicação desse instrumento teve por objetivo construir um vasto panorama sobre os desempenhos dos estudantes, de todo o EF, ao lidarem com situações-problema que envolvessem as operações multiplicação e divisão. Essas situações utilizaram apenas o conjunto dos números naturais e circunscritos a unidades e dezenas. Esse diagnóstico foi a primeira ação realizada pelo projeto.

A aplicação o referido instrumento foi coletiva, por turma, mas com resolução individual, com todos os estudantes das escolas cujos professores aceitaram participar da formação continuada oferecida no âmbito do projeto. O professor de cada turma se constituiu como auxiliar de pesquisa, já que ajudou os pesquisadores na coleta desses dados. Assim, os professores ajudaram na distribuição dos instrumentos, solicitaram, sempre que necessário, silêncio dos estudantes, receberam e guardaram dentro de um envelope os testes respondidos pelos seus estudantes e zelaram para que esses não copiassem as respostas dos colegas.

\section{O instrumento diagnóstico}

O instrumento diagnóstico estava composto por 14 situações-problema, todas do campo conceitual multiplicativo, as quais estavam distribuídas em quatro eixos, a saber: seis de proporção simples; uma de proporção dupla, três de comparação multiplicativa e quatro de produto de medidas. A seguir detalharemos essas situações por eixo.

As seis situações-problema do eixo proporção simples, três situações eram da classe "um para muitos" e as outras três, da classe "muitos para muitos". A Figura 2 apresenta as seis questões, segundo a ordem referida acima. Salientamos que a numeração delas foi a mesma do instrumento.

Figura 2: seis situações-problema de proporção simples
1) JOANA SABE QUE EM UM PACOTE HÁ 6 BISCOITOS. ELA TEM 5 PACOTES. QUANTOS BISCOITOS JOANA TEM?
4) A EsCOLA RECANTO FARÁ UMA FESTA PARA 36 CONVIDADOS. EM CADA MESA FICARÃO 4 CONVIDADOS. QUANTAS MESAS A ESCOLA PRECISARÁ ALUGAR?
8) UM SUPERMERCADO FEZ UMA PROMOÇÃO: “LEVE 4 LITROS DE SUCO POR APENAS 12 REAIS". QUANTO VAI CUSTAR CADA LITRO DE SUCO? 
3) PARA FAZER 3 FANTASIAS SÃo NECESSÁRIOS 5M DE TECIDO. ANA TEM 35M DE TECIDO. QUANTAS FANTASIAS ELA PODE FAZER?

6) CAIO COMPROU 9 CAIXAS DE SUCO E PAGOU 15 REAIS. SE ELE COMPRASSE 3 CAIXAS DE SUCO QUANTO PRECISARIA PAGAR?

12) EM UMA GINCANA NA ESCOLA SABER, A CADA 3 VOLTAS CORRENDO NA QUADRA O ALUNO GANHA 4 PONTOS. ALEX DEU 15 VOLTAS CORRENDO NA QUADRA. QUANTOS PONTOS ELE MARCOU?

Podemos observar que tanto a situação-problema 1 quanto a 3 trazem embutidas a ideia de multiplicação, de aumentar. No caso da situação-problema 3, embora sua resolução envolva tanto a multiplicação como a divisão (35 divido por 5 para achar a relação escalar entre os valores em metro, multiplicando posteriormente essa razão pelo número de fantasias (3)), na verdade, por se tratar de números pequenos, o estudante pode pensar que de 5 para 35 são 7 e então aplicar esse valor, produto escalar da relação entre os metros, para as fantasias (3 X 7). Podemos representar essa situação por meio de diagrama a seguir:

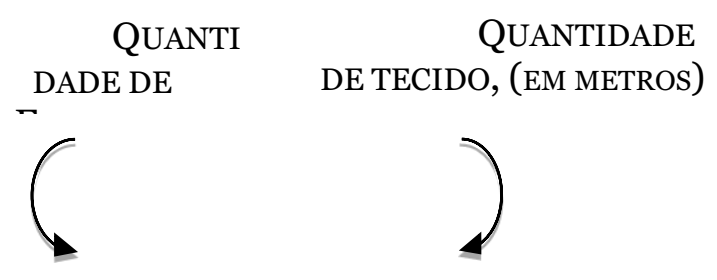

No eixo da proporção dupla, o instrumento continha uma situação-problema descrita a seguir:

Figura 3: Situação-problema de proporção dupla

14) UMA PESSOA CONSOME, EM MÉDIA, 5 LITROS DE ÁGUA EM DOIS DIAS. QUANTOS LITROS DE ÁGUA UMA FAMÍLIA COMPOSTA POR 4 PESSOAS CONSOMEM EM 6 DIAS?

Essa situação-problema foi provavelmente a mais sofisticada de todo o instrumento e pør, pelo menos, por três razões: 1) porque ela é pouco ou nada trabalhada na escola; 2) porque ela exige a realização de duas proporções simples; e, 3) sendo que seus resultados se entrelaçam no final, exigindo o produto dos dois resultados. A seguir apresentamos um esquema de uma possível solução para esta situação-problema

$\begin{array}{ccc}\text { QUANTIDADE } & \text { ÁGUA } & \text { TEMPO } \\ \text { DE PESSOAS } & \text { (EM } & \text { (EM } \\ 1 & \text { LITROS) } & \text { DIAS) } \\ 4 & 5 & 6 \\ & ? & \mathrm{X}_{3}\end{array}$

Para encontrarmos a quantidade de água consumida é preciso encontrar a proporção entre números de pessoas e consumo de água $\left(5 \mathrm{X}_{4}=20\right)$ e, na sequência encontrar a 
quantidade de água segundo o número de dias que seria $2 \times 3=6$, isto é o total de agua consumida pelas 4 pessoas, por 6 dias será de 60 litros).

No eixo de comparação multiplicativa foram três situações-problema como segue na Figura 4:

Figura 4: três situações-problema de comparação multiplicativa

2) A DiSTÂNCIA ENTRE A CASA DE LUÍS E A ESCOLA É DE 5 QUILÔMETROS E A CASA DE JOSÉ
É 4 VEZES MAIS DISTANTE. QUAL A DISTÂNCIA ENTRE A CASA DE JOSÉ E A ESCOLA?
10) CIDO TEM UMA COLEÇÃO DE 6 CARRINHOS E JOSÉ TEM UMA COLEÇÃO DE 24 CARRINHOS.
QUANTAS VEZES A COLEÇÃO DE CIDO É MENOR DO QUE A DE JOSÉ?
13) ONTEM TONHO TINHA 18 FIGURINHAS. E HOJE ELE TEM 3 VEZES MENOS. QUANTAS
FIGURINHAS ELE TEM HOJE?

É possível notar que, enquanto a situação-problema 2 requer uma operação de multiplicação, as outras duas requerem de divisão. Essas duas situações têm, por sinal um nível maior de complexidade, que é a incongruência entre as palavras do enunciado dos problemas (vezes, mais, maior) e a operação que leva a resolução correta do problema (operação de divisão).

No eixo produto de medidas foram quatro situações-problema, sendo que duas ( 5 e 7) foram elaboradas no âmbito da configuração retangular (área) e as outras duas (9 e 11) abordaram a combinatória, conforme Figura 5 a seguir.

Figura 5: quatro situações-problema de produto de medida

5) RUTE QUER MUDAR O PISO DO QUARTO DELA. ESTE QUARTO TEM 3M DE LARGURA E 6M DE COMPRIMENTO. QUANTOS METROS QUADRADOS, DE PISO, RUTE PRECISA COMPRAR?

7) A ÁREA do JARDim da CASA DE VERA É RETANGULAR E TEM 24M². A LARgura É 4M. QUAL É COMPRIMENTO EM METROS DESSE JARDIM?

9) A Lanchonete do ERnani vende 15 TiPos de SANDUíches. PARA CADA SANDUíche É USADO APENAS UM TIPO DE PÃO E UM TIPO DE RECHEIO. TEM 3 TIPOS DE PÃO (LEITE, INTEGRAL E FRANCÊS). QUANTOS TIPOS DE RECHEIO SÃO NECESSÁRIOS PARA FAZER TODOS OS TIPOS DE SANDUÍCHES?

11) NA AULA DE DANÇA DE FORRÓ TINHA 6 RAPAZES (AlEX, BETO, CAIO, DAVI, EDU, IVO) E 4 MOÇAS (MARI, FABI, LARA, SUZI). TODAS AS MOÇAS DANÇARAM COM TODOS OS RAPAZES. QUANTOS CASAIS DIFERENTES FORAM FORMADOS?

Observamos que tanto nas situações de configuração retangular, quanto de combinatória há uma situação que informa sobre as duas partes e solicita o todo e outra em que é informado sobre uma parte e sobre o todo e se pede a outra parte. As situações-problema 5 e 11 requerem uma operação de multiplicação para encontrar seu resultado, e as situações 7 e 9 são solucionadas por meio de uma operação de divisão. 
Após a apresentação do instrumento base para a realização do Estudo $\alpha$, passaremos a descrever o Estudo $\beta$.

Cabe ressaltar que a proposta idealizada para a implementação do projeto foi a seguinte: primeiro aplicaríamos, com a ajuda dos professores de cada turma, o instrumento diagnóstico com todos os estudantes da escola, incluindo os dois períodos de aula. Em seguida os dados seriam analisados pelos pesquisadores quantitativamente, em termos de desempenho, levando em consideração o ano escolar e, também, a relação, eixo e classe a que cada situação-problema pertencia, segundo o esquema proposto por Magina, Santos e Merlini (2014, 2016). Por fim, a formação teria início, tendo por base metodológica a espiral RePARe (MAGINA, 2008), discutida anteriormente. E, assim, descreveremos o Estudo $\beta$, o qual apoiou-se nos resultados do Estudo $\alpha$.

O Estudo $\beta$ teve como sujeitos os professores, em cujas escolas foi aplicado o instrumento diagnóstico e que participaram da proposta de formação de professores, utilizando o modelo metodológico da Espiral RePARe.

Esse modelo metodológico de formação foi elaborado e testado pela primeira vez por Magina em 2008 dentro do projeto (re)significar as estruturas multiplicativas a partir da formação 'reflexão-ação-planejamento-reflexão' do professor e se mostrou bastante eficiente na formação dos professores. Ele voltou a ser utilizado em parte no projeto de Santana (2009), também com sucesso. Tais resultados estimularam a voltar a utilizá-lo, agora dentro do projeto PEM (MAGINA, 2013) com o intuito de validar tal modelo.

Esta metodologia propõe que a formação se dê dialeticamente, oscilando entre três componentes: a prática docente, os resultados obtidos pelos estudantes em um diagnóstico e uma teoria da Educação Matemática que sirva de base para o entendimento dos outros componentes da formação. A sigla RePARe quer dizer: Reflexão, Planejamento, Ação, Reflexão e esses elementos se repetem, nessa ordem, ao longo de toda a formação, num formato de espiral.

Em um primeiro momento da formação, parte-se de uma reflexão teórica sobre o desempenho global dos estudantes e, a partir de então, foca-se em um determinado eixo do instrumento. Nesse momento discute-se com mais propriedade os erros e acertos dos estudantes, trazendo a tona, sempre que pertinente, explicações pautadas na Teoria dos Campos Conceituais (TCC), em especial as Estruturas Multiplicativas (VERGNAUD, 1983, 2009) como base e adotando releitura para a classificação de problemas multiplicativos lineares, feita por Magina e Cols. (MAGINA, MERLINI, SANTOS, 2014; MAGINA, SANTOS, MERLINI, 2016). O segundo momento da formação é dedicada à elaboração, por parte dos professores em formação, de duas situações-problema análogas e adaptadas para cada ano 
escolar. Essas situações-problema são planejadas dentro de um plano de aula que permita trabalhar tais situações. Na sequência os professores têm 15 dias para realizar esse planejamento em sua sala de aula, isto é, realizar a ação referente ao planejamento.

O próximo encontro de formação iniciava-se com os professores em formação apresentando e discutindo como se deu o desenvolvimento das atividades planejadas.

Dentro desse encontro, o próximo passo era apresentar aos professores o desempenho dos estudantes, dos diversos anos, em outro eixo de situações-problema do instrumento diagnóstico. Essa apresentação, mais uma vez, veio acompanhada de uma reflexão teórica, tendo a TCC e as Estruturas Multiplicativas como base. A formação continua seguindo o que propõe a espiral metodológica para formação de professores RePARe (Reflexão, Planejamento, Ação, Reflexão).

A seguir apresentamos a Espiral RePARe, que nada mais é do que a uma representação icônica que sintetiza o funcionamento dessa formação continuada de professor (Figura 6).

Observamos que a espiral é formada por uma seta que indica sua continuidade indefinidamente, isto é, o giro, composto pelos momentos de Reflexão, Planejamento, Ação, pode se repetir quantas vezes forem necessárias dentro do processo de formação. Observamos, também, que a reflexão acontece sob duas óticas, a empírica, baseada na ação do professor em sua sala de aula, e a teórica, quando a prática é discutida do ponto de vista de uma teoria (no nosso caso, a teoria dos campos conceituais). Salientamos, por fim, que a ponta da espiral é uma seta em que se encontra um sinal de reticência.

Isto significa dizer que: (a) esse caminho espiralado, em que cada volta passa necessariamente pela Reflexão (empírica e teórica), Planejamento (em pequeno e no grande grupo) e Ação na sala de aula de maneira cíclica, pode se repetir inúmeras vezes até que a proposta da formação tenha sido esgotada; e, (b) a cada volta da espiral é esperado que o conhecimento vai se ampliando e ganhado profundidade. 
Figura 6: Modelo atual da espiral RePARe, adaptada da $1^{\text {a }}$ espiral RePARe Magina (2008).

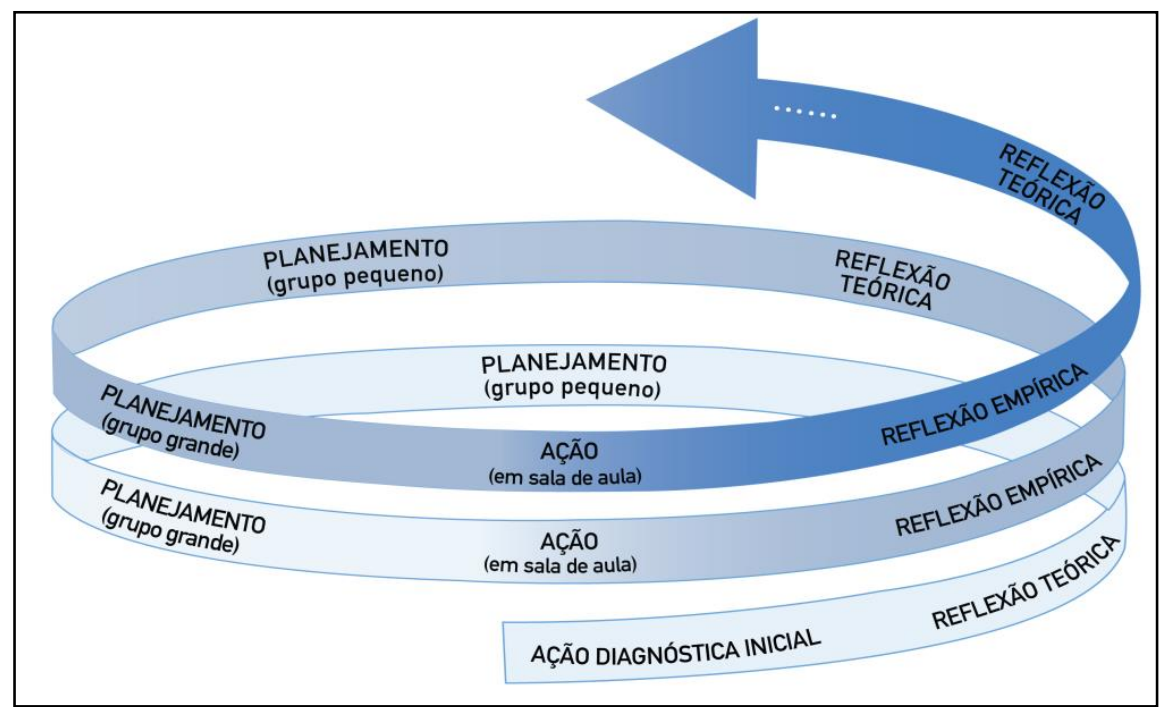

Fonte: Magina (2008).

\section{Considerações finais}

O projeto de pesquisa "As estruturas multiplicativas e a formação de professores que ensinam matemática na Bahia" - PEM se constituiu numa rede de pesquisa dentro do estado da Bahia, num elo entre pesquisadores da área de Educação Matemática que atuam em universidades baianas e grupos de pesquisa. Esse projeto consolida um modelo de formação denominado RePARe e que contribui positivamente para a formação continuada do professor e, consequentemente, para a aprendizagem do estudante; tendo uma vertente reflexiva sobre elementos que compõem o processo de ensino, bem como processos de aprendizagens dos estudantes.

Contribuição positiva, também, pode ser constatada por meio do trabalho em grupo desenvolvido pela equipe de professores dentro da própria escola, com o apoio da equipe de pesquisadores. Os momentos de estudo e reflexão sobre os resultados do diagnóstico dos estudantes e do trabalho desenvolvido em sala de aula.

Nesse cenário o modelo formativo RePARe pode ser usado para o trabalho com diferentes conteúdos matemáticos e em diferentes níveis de ensino.

Parte dos resultados dos estudos realizados no âmbito do PEM serão apresentados nos demais artigos dessa revista.

\section{Referências}

GITIRANA, V.; CAMPOS T., MAGINA, S.; SPINILLO, A. Repensando Multiplicação e Divisãao. São Paulo: PROEM Ed., 2013. 
MAgina, S. (Re)significação das Estruturas Multiplicativas. Projeto de Pesquisa. Brasília: CNPq, Edital Universal, 2008.

A pesquisa na sala de aula de matemática das séries iniciais do Ensino Fundamental: Contribuições teóricas da psicologia. Educar em Revista: UFPR, 2011, pp. 63-76.

As estruturas multiplicativas e a formação de professores que ensinam matemática na Bahia. Projeto de Pesquisa. Salvador: FAPESB, PES19/2013, 2013

MAGINA, S.; SANTOS, A.; MERLINI, V. Quando e Como devemos introduzir a divisão nas séries iniciais do Ensino Fundamental? Contribuição para o debate. EM Teia: UFPE, 2010, pp. 1-23.

MAGINA, S.; MERLINI, V.; SANTOS, A. O raciocínio de estudantes do Ensino Fundamental na resolução de situações das estruturas multiplicativas. Ciência \& Educação: UNESP, Bauru, 2014, pp. 517-533.

MAGINA, S.; MERLINI, V.; SANTOS, A.. A estrutura multiplicativa a luz da teoria dos campos conceituais: uma visão com foco na aprendizagem In: CASTRO Filho et al. Matemática, Cultura e Tecnologia: perspectivas internacionais. Curitiba: CRV, 2016, p.66-82

MORO, M.L. Estruturas multiplicativas e tomada de consciência: repartir para dividir. Psicologia: Teoria e Pesquisa [online]. 2005, vol.21, n.2, pp.217-226.

SANTANA, E. Um estudo sobre o domínio das Estruturas Aditivas nas séries iniciais do Ensino Fundamental no Estado da Bahia. Projeto de Pesquisa. Salvador: FAPESB, Projetos de Investigação Científica, 2009.

Estruturas Aditivas: o suporte didático influencia a aprendizagem do estudante? Tese (Doutorado em Educação Matemática). Pontifícia Universidade Católica de São Paulo. 2010.

SANTANA, E.; CORREIA, D. (Org.). Ensinando adição e subtração: experiências de professores de $2^{\circ}$ ano. Itabuna, BA: Via Litterarum, 2011.

SANTANA, E.; LAUTERT, S.; CASTRO FILHO, J. (Org.). Ensinando multiplicação e divisão: $4^{\circ}$ e $5^{\circ}$ anos. Itabuna, BA: Via Litterarum, 2017.

SANTOS, A. Formação de professores e as estruturas multiplicativas: reflexões teóricas e práticas. Curitiba: Ed. Appris, 2015.

VERGNAUD, G. Multiplicative structures. In: LESH, R.; LANDAU M. (Eds.). Acquisitions of mathematics concepts and procedures. New York: Academic Press, 1983, pp.127-174.

Multiplicative structures. In. Hiebert, H. and Behr, M. (Ed.). Research Agenda in Mathematics Education. Number Concepts and Operations in the Middle Grades. Hillsdale, N.J.: Lawrence Erlbaum, p. 141-161, 1988.

Multiplicative conceptual field: what and why? In: Guershon, H. e Confrey, J. (Eds.). The development of multiplicative reasoning in the learning of mathematics. Albany, N.Y.: State University of New York Press, pp. 41-59, 1994. 
O que é aprender? In: BITTAR, Marilena. MUNIZ, Cristiano Alberto (Org.). A aprendizagem matemática na perspectiva da teoria dos campos conceituais. Curitiba: Editora CRV, 2009.

\section{Agradecimentos}

A autora Sandra Magina agradece os importantes apoios recebidos pelas agências FAPESB (por meio do termo de outorga PESoo19/2013) e CNPq (por meio da bolsa Pq)

\section{Biografia Resumida}

Sandra Maria Pinto Magina - Bacharel em Psicologia pela UFPE; Mestre em Psicologia Cognitiva pela UFPE; doutora em Educação Matemática pela London University; pós-doutora em Educação Matemática pela Universidade de Lisboa. Professora Adjunta da Universidade de Santa Cruz (UESC-BA), docente dos programas de Pós-Graduação em Educação Matemática e em Educação da UESC; Líder do grupo de Pesquisa RePARe.

Link do Lattes: http://lattes.cnpq.br/8948168068305523.

e-mail: sandramagina@gmail.com

Eurivalda Ribeiro dos Santos Santana - Graduada em Ciências Matemática pela Federação das Escolas Superiores de Ilhéus e Itabuna. Mestre em Matemática pela Universidade Federal da Bahia (UFBA). Doutora em Educação Matemática pela Pontifícia Universidade Católica de São Paulo (PUC/SP). Pós-doutorado em Didática da Matemática pela Universidade de Lisboa. Professora Titular na Universidade Estadual de Santa Cruz (UESC/BA). Líder do grupo de pesquisa em educação matemática, estatística e em ciências (GPEMEC).

Link do Lattes: http://lattes.cnpq.br/7240586669577145

e-mail: eurivalda@uesc.br

Irene Mauricio Cazorla - Graduada em Estatística pela Universidad Mayor de San Marcos, Lima-Peru, mestre em Estatística e Doutora em Educação pela Universidade Estadual de Campinas (Unicamp) e Pós-doutora em Educação Matemática pela Pontifícia Universidade Católica de São Paulo (PUC/SP). Professora Plena na Universidade Estadual de Santa Cruz (UESC/BA). Líder do grupo de pesquisa em educação matemática, estatística e em ciências (GPEMEC). 
Link do lattes: http://lattes.cnpq.br/4693665009250751 e-mail: icazorla@uol.com.br. 